\title{
eJRIEPS
}

Ejournal de la recherche sur l'intervention en éducation physique et sport

Numéro spécial $2 \mid 2019$

Actes du congrès ARIS 2016

\section{Faire apprendre les élèves en milieu difficile. Analyse didactique des gestes d'un enseignant d'Éducation Physique et Sportive}

To teach students in a difficult environment. Didactic analysis of the gestures of a Physical Education teacher

Teddy Mayeko

\section{OpenEdition}

Journals

Édition électronique

URL : http://journals.openedition.org/ejrieps/950

DOI : 10.4000/ejrieps.950

ISSN : 2105-0821

\section{Éditeur}

ELLIADD

Référence électronique

Teddy Mayeko, « Faire apprendre les élèves en milieu diffıcile. Analyse didactique des gestes d'un enseignant d'Éducation Physique et Sportive », eJRIEPS [En ligne], Numéro spécial 2 | 2019, mis en ligne le 01 juillet 2019, consulté le 01 août 2019. URL : http://journals.openedition.org/ejrieps/950 DOI : 10.4000/ejrieps.950

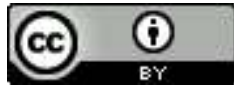

La revue eJRIEPS est mise à disposition selon les termes de la Creative Commons Attribution 4.0 International License. 


\section{Faire apprendre les élèves en milieu difficile.}

\section{Analyse didactique des gestes d'un enseignant d'Éducation Physique et Sportive.}

Teddy Mayeko*

* Laboratoire ADEF, EA 4671, Aix-Marseille Université, France.

\section{Résumé}

Nous cherchons à comprendre comment un enseignant d'Éducation Physique et Sportive (EPS) exerçant en milieu difficile peut conduire l'étude dans le but de faire apprendre tous les élèves. Dans cette optique, nous analysons ses gestes didactiques de métier en fonction de la position scolaire des élèves dans l'activité (en réussite ou en difficulté). Les résultats montrent que la proposition d'un aménagement souple et différencié du milieu didactique, la mise en place de conditions d'apprentissage favorisant la réussite des élèves et l'agencement de diverses structures de communication, contribuent à repositionner la question des savoirs au cœur du processus d'enseignement et offrent des perspectives d'apprentissage consistantes.

Mots clés : inégalités, différenciation didactique, faire apprendre, milieu difficile, savoirs

To teach students in a difficult environment. Didactic analysis of the gestures of a Physical Education teacher

\section{Summary}

We're wondering how didactic activities created by a P.E. teacher transmit actual knowledge in a socially difficult context. In order to do that, we analyse didactic gestures according to various pupils' profiles. Results show the importance of 1) implementing a variable didactic environment 2) adopting a posture towards actual success and 3) implementing various communication structure. Those three aspects help focus on knowledge in the educational process and stimulate the teaching-learning process into the group of learners.

Keys words: Inequalities, didactic differentiation, learning, difficult environment, knowledge 
Comme indiqué dans le rapport du Conseil National d'Évaluation du Système Scolaire (CNESCO) sur l'enseignement en milieu populaire (2016), les politiques éducatives accusent aujourd'hui un bilan négatif qui met en lumière l'aggravation des inégalités de traitement, de résultats, d'orientation, d'accès au diplôme ou même d'insertion professionnelle (Goussé \& Ledonné, 2016). Pour preuve, le croisement des données nationales et internationales sur l'éducation prioritaire ${ }^{1}$ en France et dans les autres pays de l'OCDE montre que pour trois domaines directement impliqués dans la réussite scolaire - à savoir le temps d'apprentissage effectif (Moignard, 2014), les pratiques pédagogiques (Bautier, 2016 ; Rochex, 2016) et l'expérience professionnelle des enseignants (Rouve \& Ria, 2008 ; Rayou \& Ria, 2009 ; Ria, 2009) - les élèves issus de milieux populaires ne bénéficient pas des mêmes conditions d'apprentissages que leurs camarades inscrits dans des établissements ordinaires. Ce constat sévère, nous alerte à la fois sur la production des inégalités scolaires - croissantes au fil de la scolarité (Felouzis, Fouquet-Chauprade, Charmillot \& Imperiale-Arefaine, 2016) - et sur les conditions de travail des enseignants qui s'avèrent extrêmement pénibles (Armand, 2011 ; Rayou, 2014 ; Bongrand \& Rochex, 2016). A titre d'exemple, l'enquête TALIS (2013) révèle qu'en éducation prioritaire la difficulté à maintenir les élèves dans une posture de travail induit une réduction importante du temps d'apprentissage. Plus concrètement, sur une année scolaire de 36 semaines, le CNESCO (2016) estime que sept semaines et demi ne sont pas consacrées à l'apprentissage des élèves, mais plutôt au contrôle de la classe.

\section{Une difficulté à enrôler les élèves dans des tâches d'apprentissage}

Les travaux consacrés à l'enseignement en milieu difficile montrent que la majorité des enseignants est confronté à un public plutôt réfractaire aux apprentissages scolaires (Van Zanten, 2001). Aussi, les missions que ces derniers doivent assumer quotidiennement sont souvent décrites comme pénibles et extrêmement fatigantes (Ria, 2009). A ce propos, l'enquête TEQ (2008), menée par le syndicat général de l'éducation nationale, révèle que $71 \%$ des jeunes enseignants de collège ont déjà consulté un médecin pour cause de fatigue due au travail. Selon Ria (2009), ce sentiment d'épuisement s'explique en partie par le

\footnotetext{
${ }^{1}$ La politique mise en œuvre à travers l'éducation prioritaire vise à corriger l'impact néfaste des inégalités sociales et économiques sur la destinée scolaire. Ainsi, l'éligibilité d'un établissement au sein du réseau de l'éducation prioritaire est calculée sur la base de quatre paramètres de difficulté sociale dont on sait qu'ils ont un impact sur la réussite scolaire (le taux de professions et catégories socioprofessionnelles défavorisées ; le taux d'élèves boursiers; le taux d'élèves résidants en zone urbaine sensible; le taux d'élève en retard à l'entrée en 6 ème).
} 
caractère très contraignant du métier. En effet, dans les établissements sensibles on note généralement une forte agitation des élèves, nécessitant en retour des efforts importants pour prendre ou reprendre le contrôle de la classe (Rouve \& Ria, 2008). Sur ce point, Debarbieux et Fotinos (2010) signalent que le fait d'enseigner en éducation prioritaire est un facteur aggravant pour tous les types de "victimation » dont les enseignants sont la cible. Pour preuve, une enquête conduite par la Direction de l'Évaluation de la Prospective et de la Performance (DEPP, 2013) montre que dans le second degré, les enseignants exerçant en éducation prioritaire sont 4 fois plus nombreux à se déclarer victimes de violence. Ces chiffres abondent concrètement dans le sens d'une détérioration des conditions de travail (Goussé \& Ledonné, 2016 ; Rochex \& Crinon, 2011 ; Van Zanten, 2001) pouvant d'ailleurs être identifiée comme une cause d'usure morale renforcée par un sentiment d'échec et d'inutilité sociale (Lantheaume \& Helou, 2008). Cela peut amener certains enseignants à proposer aux élèves : a) des tâches souvent moins ambitieuses et simplifiées (Rayou \& Sensevy, 2014) ; b) des contenus de savoir plus flous et fragmentés (Kherroubi \& Rochex, 2004 ; Marlot \& Toullec-Théry, 2011) ; ou encore c) des processus de guidage centrés en priorité sur la réussite effective (Amade-Escot \& Venturini, 2009). Tous ces modes de fonctionnement pédagogiques, consistant à négocier des exigences de formation en mettant au second plan les savoirs, génèrent des tensions importantes chez les enseignants (Espinassy \& Saujat, 2003 ; Monnier \& Amade-Escot, 2009) et participent à renforcer les inégalités scolaires au sein de la classe (Bautier, 2016 ; Rochex, 2016).

\section{Problématique de recherche}

Ainsi, dans notre étude nous cherchons à comprendre comment un professeur d'EPS, exerçant en éducation prioritaire, peut favoriser l'accès aux apprentissages. Plus spécifiquement, nous souhaitons identifier les gestes qu'il met au jour pour transmettre des contenus de savoir aux élèves tout en conciliant les diverses contraintes pesant sur son activité. De quelles ressources dispose-t-il et comment peut-il enrôler les élèves dans des enjeux de savoir consistants ? Quelle est la fonction de ses gestes didactiques et en quoi lui permettent-ils de différencier l'étude en fonction des divers besoins des élèves ? Enfin, comment peut-il réaliser ses ambitions dans un espace de travail normé par les prescrits institutionnels et l'activité concurrente de certains élèves ?

\section{Cadre théorique}




\subsection{Un double ancrage pour interroger les gestes de l'enseignant}

Dénommés selon les approches «gestes du professeur " (Chevallard, 1997), "gestes d'enseignement 》 (Sensevy, 2011), ou « geste professionnels " (Alin, 2010 ; Bucheton \& Soulé, 2009), les gestes renvoient à des techniques spécifiques (Jorro, 2002) visant à conduire l'étude au sein d'une institution donnée. Actuellement, l'ensemble des recherches portant sur les gestes montre que leur agencement joue un rôle important dans la transmission des savoirs en classe. Véritables emblèmes du travail enseignant, ils se particularisent en fonction du contexte, mais également en fonction des buts subjectifs de l'enseignant (Goigoux, 2007), des prescriptions officielles (Brière-Guenoun, 2017 ; Saujat, 2010) et des gestes d'étude mobilisés par les élèves (Bucheton \& al, 2009 ; Chevallard, 1997 ; Sensevy \& Mercier, 2007). Aussi, à partir d'un double ancrage théorique, nous prendrons appui sur le modèle de l'action conjointe en didactique (Amade-Escot, 2007 ; Sensevy \& Mercier, 2007) et sur les fondements de l'ergonomie, tels que développés en clinique de l'activité (Clot, 1999), afin de comprendre dans quelle mesure les gestes de l'enseignant permettent d'accompagner les élèves dans leurs apprentissages. Ce rapprochement nous semble fécond pour investiguer de nouveaux modèles d'analyse de l'activité professorale ayant pour but d'articuler des visées compréhensives et formatives de la recherche.

Ainsi, la première approche postule que les interactions entre le professeur et les élèves influencent la co-construction des savoirs au cours des situations d'enseignement (Mercier, Schubauer-Leoni \& Sensevy, 2002). Elle a notamment pour ambition de rendre intelligible les actions didactiques mises au jour par l'enseignant pour conduire l'étude en classe (Sensevy \& Mercier, 2007).

La seconde considère que le réel de l'activité intègre un ensemble de possible (réalisés ou non) plus large que le déroulement présent de l'action. Ces différentes catégories de possible, conceptualisées par Clot (1999) en tant qu'activité contrariée, suspendue ou empêchée, continuent d'œuvrer dans les situations d'enseignement et sont accessibles à travers la manifestation des gestes de l'enseignant (Brière-Guenoun, 2017). De fait, l'approche ergonomique nous permet d'analyser l'activité didactique tout en accédant aux possibles occultés qui pèsent sur la situation et sur le sujet. Elle traduit le plus souvent un décalage entre les prescriptions institutionnelles (ce que l'enseignant doit faire), les buts subjectifs (ce que l'enseignant veut faire) et l'activité effective (ce que l'enseignant fait) en 
révélant l'existence de tensions chez les professionnels (Brière-Guenoun, 2017). Dès lors, «si les approches didactiques se focalisent sur les liens entre les savoirs étudiés et les pratiques culturelles qui les légitiment, les approches ergonomiques analysent l'activité réelle des sujets dans des tâches prescrites qu'elles redéfinissent au regard des dimensions subjectives, sociales, institutionnelles et culturelles » (Brière-Guenoun, 2018, p.3).

\subsection{Les gestes du professeur}

D'après Sensevy et Mercier (2007), les interactions entre l'enseignant et les élèves s'organisent autour de quatre types d'actions contribuant à caractériser l'activité de l'enseignant et à révéler le développement de ses gestes. Elles consistent respectivement à : a) définir les tâches mises à l'étude, c'est à dire à transmettre les règles constitutives du jeu en permettant au professeur de poser les objets, les enjeux et les conditions de l'apprentissage ; b) dévoluer aux élèves la responsabilité de jouer le jeu de manière adéquate. En d'autres termes, cette action leur permet de s'emparer des principes définitionnels de la tâche ; c) réguler le comportement des élèves au cours de l'étude afin de les guider et les faire progresser dans les tâches ; et d) institutionnaliser les savoirs et les pratiques légitimes afin que les acteurs de la relation didactique puissent construire une référence commune et partagée. Au cours de notre étude, nous chercherons à identifier les gestes de l'enseignant en plaçant la focale sur les quatre éléments présentés ci-dessus. Cela nous permettra de comprendre comment le professeur s'y prend pour orienter les élèves vers des enjeux de savoir et comment il renforce ou stabilise la construction des compétences au sein de la classe.

\subsection{Vers les gestes didactiques de métier}

L'analyse de ces quatre types d'action vise à mieux comprendre comment le professeur agit pour diriger l'étude en classe (Sensevy \& Mercier, 2007). Or, elle peut être enrichie par un travail approfondi sur le réel de l'activité qui cible les dimensions factuelles et subjectives du travail de l'enseignant (Saujat, 2010). Grâce au mécanisme d'auto-confrontation, introduit par une méthodologie d'entretien indirecte (Clot, 1999), nous pouvons revenir sur le synopsis des leçons afin d'amener l'enseignant à décrire les possibles occultés qui pèsent sur son activité. En conséquence, l'articulation d'un modèle didactique et d'une approche ergonomique (Venturini, 2012) semble tout à fait porteuse pour étudier l'influence du métier et des prescriptions sur l'activité du professeur. En effet, d'après Kotulski et al. (2011), le 
métier peut être défini comme " une architecture entre quatre instances en tension » ( $p$. 134). Ce faisant, il est multidimensionnel (Clot, 2008) et associe les instances impersonnelle (dimension prescriptive et conceptuelle du travail), interpersonnelle (dimension collective de l'activité), transpersonnelle (dimension sociale, historique et culturelle du métier) et personnelle (dimension singulière qui fait écho au style propre de l'enseignant). Ces quatre instances sont liées structurellement et influencent l'activité de travail de l'enseignant, y compris sur le plan didactique (Brière-Guenoun, 2014).

En conséquence, nous parlerons de " gestes didactiques de métier » afin de caractériser " l'ensemble des actions spécifiques mobilisées par le professeur (définir, dévoluer, réguler, institutionnaliser) pour effectuer des tâches professorales lui permettant de conduire l'étude des élèves au sein d'une institution donnée » (Brière-Guenoun, 2017, p.42). Ces actions actualisent en contexte les buts subjectifs de l'enseignant ${ }^{2}$, eux-mêmes requestionnés par des éléments d'ordre institutionnel et culturel, tels que les prescriptions, ou les normes scolaires. Les gestes didactiques de métier s'établissent donc à partir de déterminants internes et externes pouvant être utiles pour comprendre ce qui se joue au-delà de la situation présente.

\section{Méthodologie}

\subsection{Le contexte de l'étude}

Nous avons observé les quatre premières leçons d'un cycle de tennis de table avec un enseignant débutant ( 3 années d'ancienneté) et une classe de quatrième composée de 23 élèves, dont 11 filles et 12 garçons, s'apprêtant à effectuer leur première séquence d'enseignement dans l'activité physique sportive et artistique retenue (APSA). En référence aux anciens programmes EPS de collège (2008), les élèves visent l'acquisition de la compétence attendue de Niveau $1^{3}$.

\subsection{Le dispositif de recueil des données}

\footnotetext{
2 lls sont liés notamment à ses croyances, ses valeurs et ses expériences.

3 En référence aux programmes collège de 2008, l'enseignant doit viser le niveau 1 de compétence attendue avec des élèves de 4 ème débutants dans l'activité (zéro heure de pratique effective antérieure). Notons toutefois que depuis le $1^{\text {er }}$ septembre 2016 les enseignants de collège sont soumis à de nouveaux programmes EPS qui leur offrent davantage de liberté pédagogique concernant le découpage institutionnel du temps scolaire.
} 
Le dispositif méthodologique s'inspire de celui proposé par Schubauer-Leoni et Leutenegger (2002) et des remaniements opérés pour intégrer la dimension clinique de l'activité (Brière-Guenoun, 2017). II se compose ainsi de trois phases distinctes :

- un entretien ante-cycle de type semi-directif destiné à faire émerger les intentions du professeur a priori, et notamment ses choix d'enseignement préalables, relatifs à la structuration des leçons et aux objets d'enseignement abordés ;

- des séquences vidéographiques portant sur des évènements significatifs (Leutenegger, 2009) c'est à dire des éléments factuels renseignant des modalités particulières de transmission, d'appropriation ou « d'éviction » du savoir. Elles ont été sélectionnées en fonction de leur proximité avec notre objet de recherche. Aussi, nous avons privilégié des évènements articulant conjointement les gestes didactiques de l'enseignant et les gestes d'étude des élèves afin de comprendre comment ce dernier s'y prend pour accompagner les élèves et différencier les modalités d'apprentissage.

- des entretiens post-séances, de type "autoconfrontation» (EAC) permettant de croiser les enregistrements filmés avec le point de vue du professeur.

\subsection{Le dispositif de traitement des données}

Pour chacune des séances, nous observons le type de tâche proposée et les différents types d'interactions qui émergent dans l'action conjointe entre le professeur et les élèves. Sur cette base, nous tentons de décoder les enregistrements filmés à partir d'une double analyse visant à comparer le prescrit et le réalisé (Mercier, Schubauer-Leoni \& Sensevy, 2002) :

- une analyse a priori nous permettant de définir les « contraintes et les possibles de la tâche observée " (Leutenegger, 2000). A ce propos, nous avons choisi de retenir deux épisodes susceptibles d'éclairer les modalités de transmission du savoir en contexte difficile ${ }^{4}$;

- une analyse a posteriori nous servant à identifier les gestes d'étude mobilisés par les élèves pour répondre aux tâches proposées par l'enseignant. 
Ensuite, dans le cadre des entretiens post-séances, les évènements que nous considérons comme caractéristiques de notre objet d'étude ${ }^{5}$ sont mis en lien avec les significations du professeur. Nommés « événements remarquables » (Brière-Guenoun, 2017), leur sélection renseigne des modalités particulières de transmission du savoir. Conformément aux éléments développés dans le cadre théorique, nous prenons appui sur les gestes de l'enseignant afin de saisir les manières dont il introduit, mobilise et entérine les contenus d'enseignement à des fins de transformation de l'activité des élèves et en fonction de leur niveau de pratique en tennis de table. Ces événements font ensuite l'objet d'un travail approfondi lors des entretiens d'auto-confrontation destinés à mieux comprendre la genèse de certaines tâches en lien avec les références internes et externes qui pilotent les choix du professeur. Ainsi, ce dispositif place en synergie les intentions didactiques (entretien antecycle), les contenus mis à l'étude (enregistrements filmés) et les objets de savoir assumés, remaniés, voire évacués par l'enseignant (entretiens post-séances).

\section{Résultats}

Dans cette section, nous présentons très rapidement l'échelle mésodidactique du cycle de tennis de table (c'est à dire son organisation globale) afin de revenir sur les objectifs de travail abordés par l'enseignant ${ }^{6}$. Nous en profiterons pour montrer que les manières d'agencer l'espace contribuent à optimiser l'activité d'intervention et de régulation du professeur vis-à-vis des divers élèves de la classe. Dans un second temps, nous analyserons (à l'échelle microdidactique) deux événements caractérisant les choix de l'enseignant dans la perspective de faire apprendre tous les élèves.

\subsection{Faire apprendre les élèves en organisant le travail par atelier}

Conformément aux recommandations des programmes, le cycle de tennis de table s'organise autour de la notion d'affrontement. Les élèves doivent apprendre à identifier une situation favorable de marque, pour ensuite saisir des opportunités d'action et rompre l'échange par une balle placée ou accélérée ${ }^{7}$. Ces enjeux de savoir pilotent la démarche de

$5 \quad$ Nous faisons référence aux gestes didactiques destinés à faire apprendre les élèves en milieu difficile

6 Pour plus de simplicité mais également par mesure de confidentialité, nous appellerons l'enseignant « David ».

$7 \quad$ Ce choix est piloté par la compétence attendue de niveau 1 des programmes collèges qui précise que les élèves doivent " rechercher le gain loyal d'une rencontre en assurant la continuité de l'échange, en coup 
l'enseignant et induisent des adaptations particulières en fonction du niveau de pratique des élèves, comme le révèle le synopsis (annexe 1). En effet, l'analyse a priori des divers types de tâches mis à l'étude montre que David élabore des formats pédagogiques différenciés afin de rendre accessible la culture de l'opposition à tous les élèves : "Le tennis de table est une activité d'affrontement. Ce qui compte c'est que tous les élèves soient capables d'apprendre à rompre l'échange » (entretien ante).

Ainsi, l'organisation par atelier lui permet de mettre à l'étude différents types de tâches, rattachés à des objets de savoir typiques de la compétence attendue. Plus concrètement, l'analyse a posteriori montre qu'en constituant des petits groupes David peut cibler des éléments techniques ou tactiques qui mobilisent à différents niveaux les compétences motrices et stratégiques des élèves : "Là je profite de l'organisation pour mettre en place différentes consignes en fonction de ce que je veux travailler avec les élèves. Le groupe qui est là travaille sur le placement (...), le groupe ici cherche à rompre la balle par la vitesse " (entretien post séance 3 ). L'analyse de cet extrait révèle que l'enseignant procède à une différenciation des tâches en fonction du niveau de pratique des élèves. De plus, en faisant le choix de regrouper sur une même table des élèves de même niveau, l'enseignant peut augmenter le nombre de régulations en proposant des contenus susceptibles d'intéresser tous les élèves de la zone concernée : "Ici je suis plutôt à l'aise pour passer d'une table à l'autre et essayer de donner des informations aux élèves. Le fait de les mettre comme ça me fait gagner pas mal de temps » (entretien post séance 3).

A un autre niveau, cette stratégie semble également destinée à réduire les phases de regroupement qui peuvent générer des perturbations importantes en termes de prise de parole : "Lorsque tous les élèves sont ici face à moi ils s'agitent et sont peu concentrés. Je dois les reprendre et c'est usant (...). Le fait de passer les voir à tour de rôle est beaucoup plus confortable car je peux m'adresser à trois ou quatre gamins qui vont être beaucoup plus attentifs à ce que je dis " (entretien post séance 3). Cet extrait d'EAC montre que l'enseignant est parfois empêché dans son activité et qu'il doit, le cas échéant, trouver des solutions pour réaménager son projet d'enseignement. Selon nous, ces moments d'interaction privilégiés lui permettent d'adresser aux élèves des contenus adaptés à leurs difficultés tout en minorant les interactions conflictuelles. Sur ce point, l'analyse du verbatim

droit ou en revers et en profitant d'une situation favorable pour le rompre par une balle placée latéralement ou accélérée (...) ». 
nous laisse penser que David réorganise ses gestes didactiques en fonction du caractère contraignant ou non des interactions. L'usure morale provoquée par l'inattention des élèves le conduit à envisager d'autres modalités de régulation et d'institutionnalisation des contenus.

\subsection{Faire apprendre les élèves en utilisant des objets support de différenciation}

Dans cette section, nous revenons sur un événement observé en leçon $2^{8}$. L'épisode choisi s'inscrit dans une tâche au cours de laquelle les élèves doivent apprendre à construire le plan de frappe pour assurer un renvoi sécuritaire (cf. annexe 1 : leçon 2, jouer en coup droit ou en revers). Elle cible des objets de savoir techniques, relatifs à la réalisation du coup droit et du revers (placement et trajet moteurs). Nous plaçons la focale sur un groupe d'élèves en difficulté qui réalise l'exercice avec une balle de diamètre plus important, censée favoriser la prise d'information sensori-motrice lors de la réalisation des frappes.

En tennis de table, la plasticité des objets matériels (raquette, balle et filet) permet de varier les possibilités de travail en modifiant les conditions de déroulement du jeu. Dans le cas présent, introduire une « grosse balle » au sein du milieu didactique, permet à l'enseignant d'aider les élèves les plus en difficulté à construire le renvoi. Cet aménagement didactique est en partie motivé par la nécessité d'apprendre aux élèves « à renvoyer la balle de l'autre côté du filet afin de rentrer progressivement dans une logique d'opposition " (entretien postleçon 2). Aussi, nous pouvons considérer la « grosse balle " comme une variable didactique au service de la transformation du milieu et de l'acquisition des enjeux de savoir. Plus concrètement, l'analyse a posteriori de la deuxième tâche ${ }^{9}$ (cf. annexe 1: leçon 2, coopération en coup droit/revers) révèle que son utilisation permet aux élèves les plus en difficulté de progresser dans l'identification des trajectoires de balles et dans la construction du timing de frappe. En effet, avec une balle standard les élèves les plus éloignés des attendus de la compétence ne parviennent pas à effectuer plus de 2 échanges. Raides sur leurs jambes et explosifs dans leurs frappes, les pongistes ratent fréquemment la balle et décentrent leurs frappes. A l'inverse, les élèves en réussite sont capables de jouer en

8 Le thème de la leçon porte sur la continuité de l'échange en coup droit ou en revers.

9 Cette tâche consiste pour les élèves à effectuer un plus grand nombre d'échange que les joueurs des autres tables. Les binômes d'élèves sont donc en situation de coopération à des fins de compétition. 
contrôle à une cadence relativement soutenue. Ayant déjà construit le plan de frappe et la mise à distance sur un coup préférentiel ${ }^{10}$, ils n'éprouvent pas de difficultés particulières à remettre la balle en contrôle sur l'autre demi-table. Ainsi, après avoir repris la même tâche avec une balle de diamètre supérieur, nous avons constaté de rapides progrès chez les élèves en difficulté. La lenteur du jeu doublement combiné à la grosseur de la balle et à l'accompagnement du geste au moment de la frappe permet aux élèves de réaliser plusieurs échanges au cours de la tâche. L'analyse a posteriori de ce dispositif nous amène à dire que jouer avec la "grosse balle » contribue à faciliter les prises d'informations visuelles et à réduire l'incertitude spatiale. $\mathrm{A}$ un autre niveau, cela favorise l'amplification de repères sensori-moteurs en permettant aux élèves les plus éloignés des exigences institutionnelles, de passer progressivement de frappes explosives à un accompagnement du geste au moment de la frappe. Selon David, cet aménagement permet aux joueurs en difficulté de " construire des repères plus fins et plus précis qui facilitent développement du jeu " (entretien post-leçon 2) en créant artificiellement des conditions favorables à la poursuite de l'échange.

\subsection{Faire apprendre les élèves en repensant la structure de communication}

Dans cette section, nous revenons sur un événement significatif observé en leçon $4^{11}$ (cf. annexe 1 : leçon 4 , routine plus rupture). II met au jour une structure de communication destinée à introduire les tâches auprès des élèves. Les collégiens sont deux ou trois par table et ont pour consigne de réaliser l'exercice à la suite de la présentation faite par l'enseignant. Nous cherchons ici à évaluer l'impact de ce format sur la compréhension des tâches et la mise en activité des élèves. Ainsi, au cours de notre étude nous avons pu observer deux structures communicationnelles distinctes et privilégiées lors de la définition des tâches. Une première forme de communication, dite dilogique (Leutenegger, 2008), consiste à faire passer une consigne à l'ensemble des élèves de la classe. Ce type de configuration semble empêcher l'enseignant de différencier son intervention et limite la rétention d'informations chez les élèves (Mayeko \& Brière-Guenoun, 2018). Une deuxième forme de communication dite trilogique (Leutenegger, 2008) consiste à organiser des

10 C'est généralement le revers qui nécessite moins de déplacements et de réorganisation au niveau des appuis

11 Le thème de la leçon porte sur la rupture de l'échange par une balle placée ou accélérée. 
échanges privilégiés entre trois instances : l'enseignant, un élève placé au premier plan et le reste des élèves de la classe alors considérée comme une entité collective. Comme le montre l'extrait rapporté ci-dessous, ce type de configuration permet au professeur d'expliciter les enjeux de savoirs visés au regard des dispositions spécifiques de l'élève sollicité.

David : [regroupe les élèves autour d'une table] : Bobby, mets-
toi en face de moi (...). On va engager le jeu et faire 4 échanges
[en réalité quatre passages de balles au-dessus du filet]. 1, 2, 3,
4 puis ensuite jeu libre. L'objectif pour moi c'est de marquer le
point. Bobby redit nous comment est-ce qu'on peut faire pour
marquer le point?

Bobby : On peut attaquer pour que l'adversaire ne renvoie pas la balle.

David: Exact. Vous avez bien entendu Bobby... Deux solutions : ou jattaque pour jouer rapide et laisser peu de temps à l'adversaire, ou je place pour essayer de l'éloigner de la balle. Le but c'est vraiment de prendre l'initiative pour essayer de gagner le point [il se met en place et joue selon les règles établies avec Bobby. Lorsque la balle est perdue il arrête le jeu]. C'est compris?

La classe : Oui

David : Ok, alors on y va. Pour les tables 1 à 4 on essaye plutôt d'attaquer en smashant. Pour les autres tables, essayez davantage de déplacer votre adversaire sur les côtés.

L'analyse a posteriori de cet événement montre que David met en place une nouvelle structure de communication permettant à l'ensemble des élèves de la classe de mieux se rendre compte de "ce qu'il y a à faire " au cours de la tâche. En questionnant Bobby, l'enseignant définit la tâche de façon plus interactive et renseigne les élèves sur les modalités de travail investiguées. Selon nous, cette organisation est significative en EPS car elle consiste à produire, par le biais de la démonstration, une référence en actes. Par la suite, David intervient de façon ciblée sur la motricité des élèves et moins sur les contraintes structurelles de la tâche. A titre d'exemple, il régule l'activité de Fatoumata sur son placement par rapport à la balle lors d'une attaque en smash ou celle de John sur la nécessité d'ajuster son orientation de tamis au moment de la frappe. En d'autres termes, la définition trilogique de la tâche lui permet d'optimiser son activité de régulation a posteriori. 
David s'évertue à corriger et à commenter la technique des élèves par rapport aux caractéristiques spatiales et temporelles de la frappe (la balle est derrière toi quand tu frappes, ça ne peut pas marcher; joue face à la table en revers pour bien te placer derrière la balle et mieux contrôler tes coups ; change de rythme pour attaquer, il faut que tu donnes de la vitesse à la balle). Dans cette logique, il intervient sur des aspects concrets de la motricité (placement, orientation du tamis, tenue de raquette etc.) en cherchant à faire progresser les élèves dans les phases de transition destinées à rompre l'échange. En somme, la structure de communication trilogique permet de mieux définir les enjeux de savoir et fait gagner du temps à l'enseignant lors des régulations.

\section{Conclusion}

Les résultats soulignent que les gestes de l'enseignant sont tiraillés entre d'un côté, la nécessité de faire progresser tous les élèves, et de l'autre, la difficulté à gérer leur engagement dans les tâches. Ce constat rejoint les travaux menés en ergonomie en mettant en évidence un double mouvement des prescriptions (Daniellou, 2002 ; Saujat, 2010). En effet, les auteurs distinguent les prescriptions descendantes, provenant d'une structure hiérarchique qui transmet des directives à propos des objectifs de travail visés (les injonctions institutionnelles), et les prescriptions ascendantes ou remontantes, liées à la situation de travail elle-même et provenant de trois sources différentes. La première concerne le collectif (assimilable à l'équipe pédagogique) dont on sait qu'il peut procéder à des arrangements plus ou moins importants (Brau-Antony \& Hubert, 2014). La seconde est « liée au travail réel » (Grosstephan \& Brau-Antony, 2018), et renvoie à l'activité des élèves que l'enseignant appréhende à travers son expérience personnelle. Sur ce point, les résultats montrent que David est amené à remanier son projet didactique en fonction de l'implication plus ou moins affirmée des élèves. Enfin, la dernière concerne les autoprescriptions et traduit le fait que l'enseignant personnalise, au sein de sa classe, les prescriptions externes qui lui sont adressées. Là encore, l'analyse a posteriori signale que David redéfinit les prescriptions institutionnelles en fonction de l'avancée du temps didactique et de l'interprétation qu'il fait du niveau des élèves.

De ce fait, il concilie des préoccupations liées aux contenus à enseigner (de type technique et tactique) et au contrôle de la classe (formats de travail, aménagement de l'espace et structure de communication) en s'attachant à prendre en compte les difficultés réelles des élèves. 


\section{eJRIEPS spécial 2 (juillet 2019)}

Finalement, dans cette étude, le recours aux entretiens d'auto-confrontation permet d'investiguer le réel de l'activité en mettant au jour des conflits de préoccupations sousjacents à l'activité de l'enseignant. Leur articulation avec un dispositif de type didactique, soutenu par le choix des évènements significatifs, nous amène à questionner les diverses dimensions affiliées aux gestes de l'enseignant. A la lumière des résultats produits, il semble que les buts subjectifs de l'enseignant se réactualisent dans un espace de co-ajustement aux gestes d'étude des élèves. En conséquence, David module ses gestes didactiques en tenant compte des diverses contraintes (notamment relationnelles) qui pèsent sur lui. Ainsi, les réaménagements constatés sont à la fois le signe que son activité est empêchée et qu'il procède in situ à des adaptations pertinentes pour contenir les gestes d'éviction du savoir mobilisés par les élèves.

\section{Bibliographie}

Alin, C. (2010). La Formation - Gestes professionnels et Analyse des pratiques. Paris : L'Harmattan.

Amade-Escot, C. (2007). Le didactique. Paris : éditions revue EPS,

Amade-Escot, C., \& Venturini, P. (2009). Le milieu didactique : d'une étude empirique en contexte difficile à une réflexion sur le concept. Éducation \& Didactique, 3(1), 7- 43.

Armand, A. (2011). Entretien « Les questions pédagogiques et curriculaires au cœur de la politique d'éducation prioritaire ». Revue française de pédagogie, 177, 37-46.

Bautier, E. (2016). Pratiques scolaires dominantes et inégalités sociales au sein de l'école. Contribution au rapport du Cnesco : Comment l'école amplifie-t-elle les inégalités sociales et migratoires?

Bongrand, P., \& Rochex, J-Y. (2016). La politique française d'éducation prioritaire (19812005) : les ambivalences d'un consensus, contribution préparatoire au rapport Inégalités sociales et migratoires. Comment l'école amplifie-t-elle les inégalités ? Paris : MENESR.

Brau-Antony, S., \& Hubert, B. (2014). Curriculum en Éducation Physique et Sportive et évaluation certificative au baccalauréat, Questions vives, 22, 67-82.

Brière-Guenoun, F. (2014). Instruire les gestes didactiques de métier. Contribution à un programme de recherche en didactique comparée à partir d'études menées en éducation physique et sportive. Note de synthèse pour l'Habilitation à Diriger des Recherches. Université de Toulouse, Toulouse. 
Brière-Guenoun, F. (2017). Instruire les gestes didactiques de métier. Quelles perspectives pour la formation des enseignants ? Rennes : Presses Universitaires de Rennes.

Brière-Guenoun, F. (2018). Comment penser les articulations entre approches didactiques et ergonomiques pour étudier les pratiques d'enseignement et de formation? eJRIEPS, Numéro spécial 1, 3-7.

Bucheton, D., \& Soulé, Y. (2009). Les gestes professionnels et le jeu des postures de l'enseignant dans la classe : un multi-agenda de préoccupations enchâssées. Éducation \& Didactique, 3(3), 29-48.

Chevallard, Y. (1997). Familière et problématique, la figure du professeur. Recherche en Didactique des Mathématiques, 17/3, 17-54.

Clot, Y. (1999). La fonction psychologique du travail. Paris : PUF, Le travail humain.

Clot, Y. (2008). Travail et pouvoir d'agir. Paris : Presses universitaires de France.

Conseil National d'Évaluation du Système Scolaire. (2016). Inégalités sociales et migratoires. Comment l'école amplifie-t-elle les inégalités. Rapport scientifique.

Daniellou, F. (2002). Le travail des prescriptions. Actes du 37ième Congrès de la SELF « Nouvelles formes de travail, nouvelles formes d'analyse. Aix en Provence, 25-27 Septembre.

Debarbieux, E., \& Fotinos, G. (2010). Violence et climat scolaire dans les établissements du second degré en France, une enquête quantitative auprès des personnels de direction des lycées et collèges. Observatoire International de la Violence à l'école.

Espinassy, L., \& Saujat, F. (2003). Enseigner les arts plastique en ZEP. Les dessous du métier. Recherche et formation, 44, 111-124.

Felouzis, G., Fouquet-Chauprade, B., Charmillot, S., \& Imperiale-Arefaine, L. (2016). Inégalités scolaires et politiques d'éducation. Contribution au rapport du Cnesco : Comment l'école amplifie-t-elle les inégalités sociales et migratoires?

Goigoux, R. (2007). Un modèle d'analyse de l'activité des enseignants, Éducation \& didactique, 1 (3), 47-69.

Goussé, M., \& Le Donné, N. (2016). Pourquoi les inégalités de compétences cognitives à 15 ans ont- elles tant augmenté en France ? Contribution au rapport du Cnesco : Comment l'école amplifie-t-elle les inégalités sociales et migratoires?

Grosstephan, V., \& Brau-Antony, S. (2018). Analyser l'activité évaluative des enseignants d'EPS : les apports des approches ergonomique et didactique. eJRIEPS, Numéro spécial 1, 129-145. 
Jorro, A. (2002). Professionnaliser le métier d'enseignant. Paris : ESF.

Kherroubi, M., \& Rochex, J-Y. (2004). La recherche en éducation et les ZEP en France. 2. Apprentissages et exercice professionnel en ZEP : résultats, analyses, interprétations. Revue française de pédagogie, 146, 115-190.

Kotulski, K., Clot, Y., Litim, M., \& Plateau, S. (2011). L'horizon incertain de la transformation en clinique de l'activité : une intervention dans le champ de l'éducation surveillée. Activités, 8 (1), 129-145.

Lantheaume, F., \& Helou, C. (2008). La souffrance des enseignants. Une sociologie pragmatique du travail enseignant. Paris : PUF.

Leutenegger, F. (2000). Construction d'une « clinique » pour le didactique. Une étude des phénomènes temporels de l'enseignement. Recherche en Didactique des Mathématiques, 20 (2), 209-250.

Leutenegger, F. (2008). L'entrée dans un code écrit à l'école enfantine et l'articulation entre le collectif et l'individuel : comparaison de deux études de cas. Éducation \& didactique, 2 (2), 7-42.

Leutenegger, F. (2009). Le temps d'instruire. Approche clinique et expérimentale du didactique ordinaire en mathématiques. Bruxelles : Peter Lang.

Marlot, C., \& Toullec-Théry, M. (2011). Caractérisation didactique des gestes de l'aide ordinaire à l'école élémentaire : une étude comparative de deux cas didactiques limite en mathématiques. Éducation \& didactique, 5 (3), 7-32.

Mayeko, T., \& Brière-Guenoun, F. (2018). La différenciation didactique active : entre progression individualisée et construction implicite des inégalités. In F. BrièreGuenoun, F., S. Couchot-Schiex, M-P. Poggi, \& I. Verscheure, Les inégalités d'accès aux savoirs se construisent aussi en EPS, Analyses didactiques et sociologiques (pp. 65-78). Besançon : PUFC, collection Pratiques et Techniques.

Mercier, A. Schubauer-Leoni, M-L. \& Sensevy, G. (2002). Vers une didactique comparée. Revue Française de pédagogie, 141, 5-16.

Moignard, B. (2014). Le collège fantôme. Mesurer l'exclusion temporaire des collégiens. Diversité, 175, 23-34.

Monnier, N., \& Amade-Escot, C. (2009). L'activité didactique empêchée : outil d'intelligibilité de la pratique enseignante en milieu difficile. Revue française de pédagogie, 168, 59-73. 
Rayou, P. (2014). Sociologie et didactique. Vers un espace commun de problématisation. Éducation \& didactique, 8(1), 91-100.

Rayou, P., \& Ria, L. (2009). Former les nouveaux enseignants. Autour des statuts, de l'organisation et des savoirs professionnels. Éducation et Sociétés, 23(1), 79-90.

Rayou, P., \& Sensevy, G. (2014). Milieux didactiques et contextes sociaux. Les arrières plans des apprentissages. Revue Française de Pédagogie, 188, $23-38$.

Ria, L. (2009). De l'analyse de l'activité des enseignants débutants en milieu difficile à la conception de dispositifs de formation. In M. Durand \& L. Filliettaz. La place du travail dans la formation des adultes, (pp. 217-243). Paris : PUF.

Rochex, J-Y. (2016). Les inégalités scolaires en France, des structures aux pratiques. Animation \& Éducation, 252, 12-13.

Rochex, J-Y., \& Crinon, J. (2011). La construction des inégalités scolaires. Au cœur des pratiques et des dispositifs d'enseignement. Rennes: Presses universitaires de Rennes.

Rouve, M., \& Ria, L. (2008). Analyse de l'activité professionnelle d'enseignants néotitulaires en réseau ambition réussite : études de cas. Travail \& Formation en Éducation. Consulté sur internet : http://tfe.revues.org/

Saujat, F. (2010). Travail, formation et développement des professionnels de l'éducation : voies de recherche en sciences de l'éducation. Note de synthèse pour l'Habilitation à Diriger des Recherches. Université de Provence, Aix-Marseille I.

Schubauer-Leoni, M-L. (2008). La construction de la référence dans l'action conjointe professeur-élève. In N. Wallian, M. P. Poggi \& M. Musard (Éd.), Co-construire des savoirs: les métiers de l'intervention par les APSA (pp. 67-86). Besançon : PUFC.

Schubauer-Leoni, M-L., \& Leutenegger, F. (2002). Expliquer et comprendre dans une approche clinique/expérimentale du didactique ordinaire. In F. Leutenegger \& M. Saada-Robert (Eds), Expliquer et comprendre en sciences de l'éducation. Paris, Bruxelles : De Boeck.

Sensevy, G. (2011). Le sens du savoir. Éléments pour une théorie de l'action conjointe en didactique. Bruxelles : De Boeck Université.

Sensevy, G., \& Mercier, A. (2007). Agir ensemble. L'action conjointe du professeur et des élèves dans le système didactique. Rennes : PUR.

Van Zanten, A. (2001). L'école de la périphérie. Paris : PUF. 


\section{Annexes}

\section{Annexe 1. Synopsis : Agencement des objets de savoir au sein des tâches mises à l'étude lors des quatre premières leçons du cycle.}

\begin{tabular}{|c|c|c|c|c|c|}
\hline Leçons & Objectifs visés & Type de tâche & \multicolumn{3}{|c|}{ Objets de savoir } \\
\hline \multirow[t]{3}{*}{1} & \multirow{3}{*}{$\begin{array}{l}\text { S'opposer de } \\
\quad \text { façon } \\
\text { réglementaire }\end{array}$} & Jonglage & $\begin{array}{c}\text { Contrôle de la } \\
\text { balle }\end{array}$ & $\begin{array}{l}\text { Tenue de } \\
\text { raquette }\end{array}$ & $\begin{array}{l}\text { Assurer le } \\
\text { comptage }\end{array}$ \\
\hline & & $\begin{array}{c}\text { Continuité en coup } \\
\text { droit/revers }\end{array}$ & $\begin{array}{c}\text { Contrôle de la } \\
\text { balle }\end{array}$ & Plan de frappe & $\begin{array}{c}\text { Renvoi } \\
\text { sécuritaire }\end{array}$ \\
\hline & & $\begin{array}{c}\text { Montante } \\
\text { Descendante }\end{array}$ & $\begin{array}{l}\text { Règles de } \\
\text { l'activité }\end{array}$ & $\begin{array}{l}\text { Entrer dans } \\
\text { l'opposition }\end{array}$ & $\begin{array}{c}\text { Gagner ou } \\
\text { perdre }\end{array}$ \\
\hline \multirow[t]{3}{*}{2} & \multirow[t]{3}{*}{$\begin{array}{c}\text { Jouer en coup } \\
\text { droit ou en revers }\end{array}$} & $\begin{array}{c}\text { Continuité en coup } \\
\text { droit/revers }\end{array}$ & $\begin{array}{c}\text { Contrôle de la } \\
\text { balle }\end{array}$ & Plan de frappe & $\begin{array}{c}\text { Renvoi } \\
\text { sécuritaire }\end{array}$ \\
\hline & & $\begin{array}{c}\text { Coopération en coup } \\
\text { droit/revers }\end{array}$ & $\begin{array}{c}\text { Contrôle de la } \\
\text { balle }\end{array}$ & Plan de frappe & $\begin{array}{c}\text { Renvoi } \\
\text { sécuritaire }\end{array}$ \\
\hline & & $\begin{array}{c}\text { Match en coup } \\
\text { droit/revers }\end{array}$ & $\begin{array}{c}\text { Placement } \\
\text { déplacement }\end{array}$ & Plan de frappe & Précision \\
\hline \multirow[t]{3}{*}{3} & \multirow{3}{*}{$\begin{array}{c}\text { Identifier une } \\
\text { situation } \\
\text { favorable }\end{array}$} & $\begin{array}{c}\text { Coopération en coup } \\
\text { droit/revers }\end{array}$ & $\begin{array}{c}\text { Contrôle de la } \\
\text { balle }\end{array}$ & Plan de frappe & $\begin{array}{c}\text { Renvoi } \\
\text { sécuritaire }\end{array}$ \\
\hline & & $\begin{array}{l}\text { Rupture annoncée } \\
\qquad(« \text { Banco ») }\end{array}$ & $\begin{array}{c}\text { Reconnaître un } \\
\text { contexte } \\
\text { d'attaque }\end{array}$ & $\begin{array}{l}\text { Saisir une } \\
\text { opportunité }\end{array}$ & $\begin{array}{l}\text { Placer ou } \\
\text { accélérer }\end{array}$ \\
\hline & & $\begin{array}{c}\text { Montante } \\
\text { descendante type } \\
\text { «Banco } »\end{array}$ & $\begin{array}{l}\text { Saisir une } \\
\text { opportunité }\end{array}$ & $\begin{array}{l}\text { Placer ou } \\
\text { accélérer }\end{array}$ & $\begin{array}{c}\text { Gagner ou } \\
\text { perdre }\end{array}$ \\
\hline \multirow[t]{3}{*}{4} & \multirow{3}{*}{$\begin{array}{c}\text { Rompre l'échange } \\
\text { par une balle } \\
\text { placée ou } \\
\text { accélérée }\end{array}$} & $\begin{array}{c}\text { Rupture annoncée } \\
(« \text { Banco })\end{array}$ & $\begin{array}{c}\text { Reconnaître un } \\
\text { contexte } \\
\text { d'attaque }\end{array}$ & $\begin{array}{l}\text { Saisir une } \\
\text { opportunité }\end{array}$ & $\begin{array}{l}\text { Placer ou } \\
\text { accélérer }\end{array}$ \\
\hline & & Routine + rupture & $\begin{array}{c}\text { Renvoyer sans } \\
\text { s'exposer }\end{array}$ & $\begin{array}{c}\text { Construire une } \\
\text { opportunité }\end{array}$ & $\begin{array}{l}\text { Placer ou } \\
\text { accélérer }\end{array}$ \\
\hline & & $\begin{array}{c}\text { Tournoi équipe par } \\
\text { poule }\end{array}$ & $\begin{array}{l}\text { Gagner ou } \\
\text { perdre }\end{array}$ & Coacher & $\begin{array}{l}\text { Remplir une } \\
\text { feuille de score }\end{array}$ \\
\hline
\end{tabular}

